\title{
DIVA: A Reliable Substrate for Deep Submicron Microarchitecture Design
}

\author{
Todd M. Austin ${ }^{1}$ \\ Advanced Computer Architecture Laboratory \\ University of Michigan \\ taustin@eecs.umich.edu
}

\begin{abstract}
Building a high-performance microprocessor presents many reliability challenges. Designers must verify the correctness of large complex systems and construct implementations that work reliably in varied (and occasionally adverse) operating conditions. To further complicate this task, deep submicron fabrication technologies present new reliability challenges in the form of degraded signal quality and logic failures caused by natural radiation interference.

In this paper, we introduce dynamic verification, a novel microarchitectural technique that can significantly reduce the burden of correctness in microprocessor designs. The approach works by augmenting the commit phase of the processor pipeline with a functional checker unit. The functional checker verifies the correctness of the core processor's computation, only permitting correct results to commit. Overall design cost can be dramatically reduced because designers need only verify the correctness of the checker unit.

We detail the DIVA checker architecture, a design optimized for simplicity and low cost. Using detailed timing simulation, we show that even resource-frugal DIVA checkers have little impact on core processor performance. To make the case for reduced verification costs, we argue that the DIVA checker should lend itself to functional and electrical verification better than a complex core processor. $F i$ nally, future applications that leverage dynamic verification to increase processor performance and availability are suggested.
\end{abstract}

\section{Introduction}

Reliable operation is perhaps the single most important attribute of any computer system, followed closely by performance and cost. Users need to be able to trust that when the processor is put to a task the results it renders are correct. If this is not the case, there can be serious repercussions, ranging from disgruntled users to financial damage to loss of life. There have been a number of high-profile

\footnotetext{
${ }^{1}$ This work was performed while the author was (un)employed as an independent consultant in late May and June of 1999.
}

examples of faulty processor designs. Perhaps the most publicized case was the Intel Pentium FDIV bug in which an infrequently occurring error caused erroneous results in some floating point divides [16]. More recently, the MIPS $\mathrm{R} 10000$ microprocessor was recalled early in its introduction due to implementation problems [18]. These faulty parts resulted in bad press, lawsuits, and reduced customer confidence. In most cases, the manufacturers replaced the faulty parts with fixed ones, but only at great cost. For example, Intel made available replacement Pentium processors to any customer that had a faulty part and requested a replacement, at an estimated cost of $\$ 475$ million [32].

\subsection{Designing Correct Processors}

To avoid reliability hazards, chip designers spend considerable resources at design and fabrication time to verify the correct operation of parts. They do this by applying functional and electrical verification to their designs. ${ }^{2}$

Functional Verification Functional verification occurs at design time. The process strives to guarantee that a design is correct, i.e., for any starting state and inputs, the design will transition to the correct next state. It is quite difficult to make this guarantee due to the immense size of the test space for modern microprocessors. For example, a microprocessor with 32 32-bit registers, 8k-byte instruction and data caches, and 300 pins would have a test space with at least $2^{132396}$ starting states and up to $2^{300}$ transition edges emanating from each state. Moreover, much of the behavior in this test space is not fully defined, leaving in question what constitutes a correct design.

Functional verification is often implemented with simulation-based testing. A model of the processor being designed executes a series of tests and compares the model's results to the expected results. Tests are constructed to provide good coverage of the processor test space. Unfortunately, design errors sometimes slip through this testing

\footnotetext{
2Often the term "validation" is used to refer to the process of verifying the correctness of a design. In this paper we adopt the nomenclature used in the formal verification literature, i.e., verification is the process of determining if a design is correct, and validation is the process of determining if a design meets customers' needs. In other words, verification answers the question, "Did we build the chip right?", and validation answers the question, "Did we build the right chip?".
} 
process due to the immense size of the test space. To minimize the probability of this happening, designers employ various techniques to improve the quality of verification including co-simulation [6], coverage analysis [6], random test generation [1], and model-driven test generation [13].

A recent development called formal verification [15] works to increase test space coverage by using formal methods to prove that a design is correct. Due to the large number of states that can be tested with a single proof, the approach can be much more efficient than simulation-based testing. In some cases it is even possible to complctely verify a design, however, this level of success is usually reserved for in-order issue pipelines or simple out-of-order pipelines with small window sizes. Complete formal verification of complex modern microprocessors with out-oforder issue, speculation, and large windows sizes is currently an intractable problem [8, 24].

Electrical Verification Functional verification only verifies the correctness of a processor's function at the logic level, it cannot verify the correctness of the logic implementation in silicon. This task is performed during electrical verification. Electrical verification occurs at design time and fabrication time (to speed bin parts). Parts are stress tested at extreme operating conditions, e.g., low voltage, high temperature, high frequency, and slow process, until they fail to opcrate. ${ }^{3}$ The allowed maximum (or minimum) for each of these operating conditions is then reduced by a safe operating margin (typically $10-20 \%$ ) to ensure that the part provides robust operation at the most extreme operating conditions. If after this process the part fails to meet its operational goals (e.g., frequency or voltage), directed testing is used to identify the critical paths that are preventing the design from reaching these targets [3].

Occasionally, implementation errors slip through the electrical verification process. For example, if an infrequently used critical path is not exercised during electrical verification, any implementation errors in the circuit will not be detected. Data-dependent implementation errors are perhaps the most difficult to find because they require very specific directed testing to locate. Examples of these type of errors include parasitic crosstalk on buses [4], Miller effects on transistors [31], charge sharing in dynamic logic [31], and supply voltage noise due to $\frac{d I}{d t}$ spikes [25].

\subsection{Deep Submicron Reliability Challenges}

To further heighten the importance of high-quality verification, new reliability challenges are materializing in deep submicron fabrication technologies (i.e., process technologies with minimum feature sizes below $0.25 \mu \mathrm{m}$ ). Finer feature sizes result in an increased likelihood of noise-related faults, interference from natural radiation sources, and huge verification burdens brought on by increasingly complex designs. If designers cannot meet these new reliability challenges, they may not be able to enjoy the cost and speed advantages of these denser technologies.

\footnotetext{
${ }^{3}$ Or fail to meet a critical design constraint such as power dissipation or mean time to failure (MTTF).
}

Noise-Related Faults Noise related faults are the result of electrical disturbances in the logic values held in circuits and wires. As process feature sizes shrink, interconnect becomes increasingly susceptible to noise induced by other wires $[4,23]$. This effect, often called crosstalk, is the result of increased capacitance and inductance due to densely packed wires [2]. At the same time, designs are using lower supply voltage levels to decrease power dissipation, resulting in even more susceptibility to noise as voltage margins are decreased.

Natural Radiation Interference There are a number of radiation sources in nature that can affect the operation of electronic circuits. The two most prevalent radiation sources are gamma rays and alpha particles. Gamma rays arrive from space. While most are filtered out by the atmosphere, some occasionally reach the surface of the earth, especially at higher altitudes [33]. Alpha particles are created when atomic impurities (found in all materials) decay [21]. When these energetic particles strike a very small transistor, they can deposit or remove sufficient charge to temporarily turn the device on or off, possibly creating a logic error $[11,23]$. Energetic particles have been a problem for DRAM designs since the late 1970's when DRAM capacitors became sufficiently small to be affected by energetic particles [21].

It is difficult to shicld against natural radiation sources. Gamma rays that reach the surface of the earth have sufficiently high momentum that they can only he stopped with thick, dense materials [33]. Alpha particles can be stopped with thin shields, but any effective shield would have to be free of atomic impurities, otherwise, the shield itself would be an additional source of natural radiation. Neither shielding approach is cost effective for most system designs. As a result, designers will likely be forced to adopt fault-tolerant design solutions to protect against natural radiation interference.

Increased Complexity With denser feature sizes, there is an opportunity to create designs with many millions and soon billions of transistors. While many of these transistors will be invested in simple regular structures like cache and predictor arrays, others will find their way into complex components such as dynamic schedulers, new functional units, and other yet-to-be-invented gadgets. There is no shortage of testimonials from industry leaders warning that increasing complexity is perhaps the most pressing problem facing future microprocessor designs $[5,30,14,10]$. Without improved verification techniques, future designs will likely be more costly, take longer to design, and include more undetected design errors.

\subsection{Dynamic Verification}

Today, most commercial microprocessor designs employ fault-avoidance techniques to ensure correct operation. Design faults are avoided by putting parts through extensive functional verification. To ensure designs are electrically robust, frequency and voltage margins are inserted into acceptable operating ranges. These techniques, however, are 


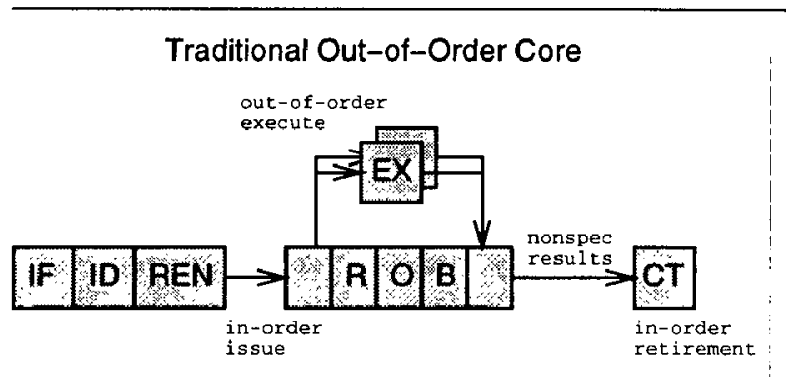

a)

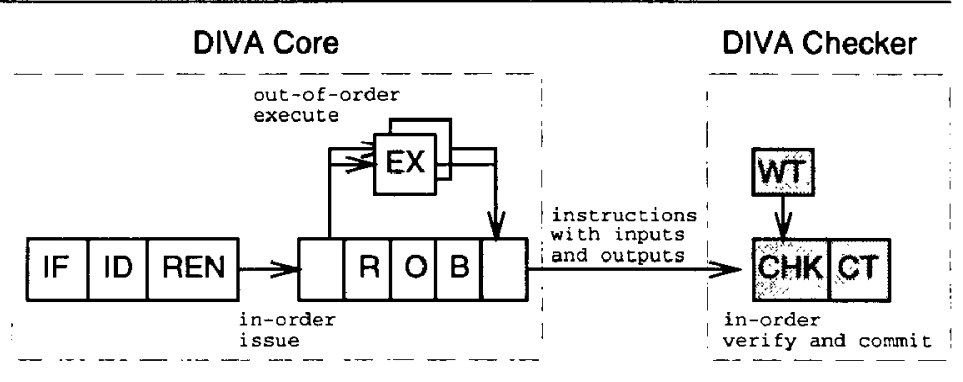

b)

Figure 1. Dynamic Verification. Figure a) shows a traditional out-of-order processor core. Figure b) shows the core augmented with a checker stage (labeled CHK). The shaded components in each figure indicate the part of the processor that must be verified correct to ensure correct computation.

becoming less attractive for future designs due to increasing complexity, degraded signal integrity, and natural radiation interference.

Traditional fault-tolerance techniques could address some of the reliability challenges in future designs, but only at great cost. System-level techniques such as triple modular redundancy (TMR) [26] can detect and correct a single transient fault in the system at the expense of three times the hardware plus voting logic. Logical-level fault-tolerant solutions, such as self-checking logic [17], often have lower cost (typically only twice as much hardware), but they are still costly and can slow critical circuits. Moreover, these approaches only address transient errors, i.e., errors which manifest temporarily (such as energetic particle strikes). They cannot address design errors if the error occurs within each of the redundant components - the redundant units will simply agree to take the wrong action.

In this paper, we introduce dynamic verification, a novel microarchitecture-based technique that permits detection and recovery of all functional and electrical faults in the processor core, both permanent and transient. A dynamic implementation verification architecture (DIVA) extends the speculation mechanism of a modern microprocessor to detect errors in the computation of the processor core. As shown in Figure 1, a DIVA processor is created by splitting a traditional processor design into two parts: the deeply speculative DIVA core and the functionally and electrically robust DIVA checker. The DIVA core is composed of the entire microprocessor design except the retirement stage. The core fetches, decodes, and executes instructions, holding their speculative results in the re-order buffer (ROB). When instructions complete, their input operands and results are sent in program order to the DIVA checker. The DIVA checker contains a functional checker stage (CHK) that verifies the correctness of all core computation, only permitting correct results to pass through to the commit stage (CT) where they are written to architected storage. If any errors are detected in the core computation, the checker fixes the errant computation, flushes the processor pipeline, and then restarts the processor at the next instruction.

Certain faults, especially those affecting core processor control circuitry, can lock up the core processor or put it into a deadlock or livelock state where no instructions attempt to retire. For example, if an energetic particle strike changes an input tag in a reservation station to the result tag of the same instruction, the processor core scheduler will deadlock. To detect these faults, a watchdog timer (WT) is added. After each instruction commits, the watchdog timer is reset to the maximum latency for any single instruction to complete. If the timer expires, the processor core is no longer making forward progress and the core is restarted. To ensure that the processor core continues making forward progress in the event of an unrecoverable design fault, the checker is able to complete execution of the current instruction before restarting the core processor.

On the surface, it may seem superfluous to add hardware to perform a verification function that is today accomplished at design time. However, there are at least four powerful advantages of dynamic verification:

- The approach concentrates functional and electrical verification into the checker unit. As a result, the core processor has no burden of functional or electrical correctness, and no requirement of forward progress. If the checker design is kept simple, the approach can reduce the cost and improve the overall quality of processor verification.

- Transistors outside of the checker unit can scale to smaller sizes without fear of natural radiation interference. If these transistors experience an energetic particle strike and produce incorrect results, the checker will detect and correct any errant computation.

- The fault-avoidance techniques used to produce electrically robust designs are very conservative. By leveraging a dynamic verification approach, voltage and timing margins in the core can be significantly tightened, resulting in faster and cooler implementations.

- Without the necessity to be correct, it becomes possible to simplify the processor core, e.g., infrequently used circuits can be eliminated to improve the speed or reduce the size of critical circuit paths.

In the remainder of this paper, we present and evaluate the DIVA checker architecture. In Section 2, we detail the 


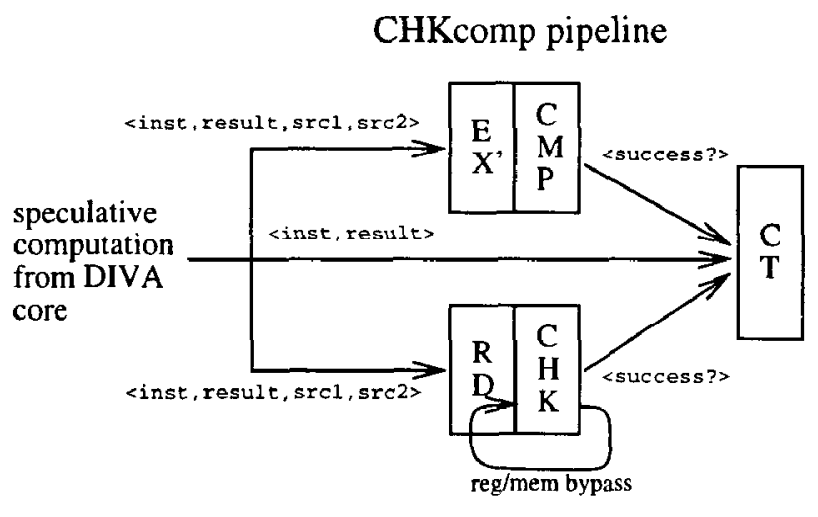

CHKcomm pipeline

a)

\begin{tabular}{|c|c|c|c|}
\hline $\begin{array}{l}\text { stage } \\
\text { inst } \\
\text { class }\end{array}$ & RD & CHK & CT \\
\hline $\begin{array}{l}\text { ALU/ } \\
\text { AGEN/ } \\
\text { BR }\end{array}$ & $\begin{array}{l}\text { read src1 } \\
\text { read } s r c 2\end{array}$ & $\begin{array}{l}\text { check src1 } \\
\text { check src2 } \\
\text { wait for } \\
\text { CHKcomp }\end{array}$ & $\begin{array}{l}\text { WB result } \\
\text { or } \\
\text { exception }\end{array}$ \\
\hline LD & $\begin{array}{l}\text { read addr } \\
\text { read mem }\end{array}$ & $\begin{array}{l}\text { check addr } \\
\text { check mem }\end{array}$ & $\begin{array}{l}\text { WB result } \\
\text { or } \\
\text { exception }\end{array}$ \\
\hline ST & $\begin{array}{l}\text { read addr } \\
\text { read st data }\end{array}$ & $\begin{array}{l}\text { check addr } \\
\text { check st data }\end{array}$ & $\begin{array}{l}\text { ST mem } \\
\text { or } \\
\text { exception }\end{array}$ \\
\hline
\end{tabular}

b)

Figure 2. A Dynamic Implementation Verification Architecture (DIVA). Figure a) illustrates the DIVA architecture and its interface to the core processor. Figure b) details the DIVA CHKcomm pipeline operation for each instruction class.

architecture and operation of the DIVA checker unit. We also present arguments why the DIVA checker should be low cost and lend itself to functional and electrical verification, more so than the complex core processor it monitors. In Section 3, we present analyses of the runtime impacts of dynamic vcrification. Through detailed timing simulation, we examine the performance impacts of various DIVA checker architectures. We also study the effect of fault rates on core processor performance. Section 4 describes related work, and Section 5 suggest other applications of dynamic verification. Finally, Section 6 gives conclusions.

\section{The DIVA Checker Architecture}

The DIVA checker architecture presented in this section makes a number of important assumptions concerning the underlying microarchitecture. First, it is assumed that all architected registers and memory employ an appropriate coding technique (e.g., ECC) to detect and correct any storagerelated faults. As a result, any value the DIVA checker reads or writes to a register or memory will complete without error. Second, it is assumed that the record of instructions fetched by the DIVA core are correctly communicated to the DIVA checker. Once again, coding techniques can be used to detect and correct errors in this communication. (Note that it is not assumed that accesses to instruction or data storage occurred in the right order or to the correct address, the DIVA checker will verify these requirements.)

\subsection{Basic Operation}

Figure 2a details the architecture of the DIVA checker. The DIVA core processor sends completed instructions in program order to the DIVA checker. With each instruction, it also sends the operands (inputs) and result (output) values as computed by the DIVA core processor. The checker ver- ifies the instruction result using two parallel and independent verification pipelines. The CHKcomp pipeline verifies the integrity of all core processor functional unit computation. The CHKcomm pipeline ensures that register and memory communication between core processor instructions occurred without error. If both pipelines report correct operations (and no earlier instruction declared an error), the core computation succeeded with the correct inputs. Hence, the instruction result is correct and it can be safely retired to architected storage in the commit stage (CT) of the pipeline.

CHKcomp Pipeline The CHKcomp pipeline verifies the integrity of all functional unit computation. In the EX' stage of the CHKcomp pipeline, the result of the instruction is re-computed. In the CMP stage, the re-computed result is compared to the functional unit result delivered by the core processor. If the two results differ, the DIVA checker raises a CHKcomp exception which will correct the errant core result with the value computed in the EX' stage.

Because instructions are delivered by the DIVA core to the CHKcomp pipeline with pre-computed inputs and outputs, there are no inter-instruction dependencies to slow pipeline progress. In addition, bypass datapaths are not required, and pipeline control logic is trivial, making the CHKcomp pipeline simple and fast.

It may seem redundant to execute the instruction twice: once in the functional unit and again in the CHKcomp pipeline, however, there is good reason for this approach. First, the implementation of the CHKcomp pipeline can take advantage of a simpler algorithnn to reduce functional unit verification costs. Second, the CHKcomp pipeline can be implemented with large transistors (that carry ample charge) and large timing and voltage margins, making it resistant to natural radiation interference and noise-related faults. 
CHKcomm Pipeline Figure $2 b$ details operation of the CHKcomm pipeline for each instruction class. For the purpose of demonstration, it is assumed that the underlying architecture is a simple load/store instruction set (although this is not required). In addition, load and store operations are decomposed into two sub-operations: an address generation operation (AGEN) which adds a register and constant to produce an effective address, and a corresponding load (LD) or store (ST) primitive that accepts the effective address. This decomposition simplifies the mapping of load and store operations onto the CHKcomm pipelinc.

The CHKcomm pipeline verifies that the processor core produced the correct register and memory input operands for each instruction. It does this by re-executing all communication in program order just prior to instruction retirement. In the RD stage of the CHKcomm pipeline, the register and memory operands of instructions are read from architected storage. In the CHK stage of the pipeline, these values are compared to the input values delivered by the core processor. If the operands delivered by the core processor match those read by the RD stage, the processor core successfully implemented instruction communication and processing may continue. Otherwise, the DIVA checker raises a CHKcomm register or memory exception which will restore the correct input operand with the operand value read in the RD stage.

Like the CHKcomp pipeline, the DIVA core delivers instructions to the CHKcomm pipeline with pre-computed inputs and outputs. This eliminates any inter-instruction dependencies that can stall the flow of instructions. A single bypass exists across the CHK stage to handle the case of an instruction checking an input written by the immediately previous instruction. Since this value is not visible until the CT stage of the pipeline (when the value is written to architected state), a single bypass is provided to eliminate any stalls.

It may seem superfluous to re-execute all storage operations in the CHKcomm pipeline, especially given the assumption that all storage is protected from faults using coding techniques such as ECC. However, coding techniques are insufficient to detect all communication errors. While coding can detect storage values that were damaged by transient errors, it cannot detect failed or misdirected communication in the processor core. For example, if the register renamer points an operand to an incorrect physical storage location, or if the store forward buffers miss a communication through aliased virtual memory, coding techniques will not detect these errors. These errors are, however, detected by the CHKcomm pipeline as it re-executes register and memory communication. Instruction fetch accesses, on the other hand, do not need to be re-executed because the order of accesses to this storage is not important (save selfmodifying code writes).

\subsection{Fault Handling}

In the event a fault is detected in the DIVA core computation, the DIVA checker will raise an exception to correct the errant condition and restart the processor. Figure 3 shows the DIVA exceptions that can occur, their priority, and the specific method used to recover machine state.

Exceptions are handled in program order at the commit (CT) stage of the pipeline. If an instruction declares multiple exceptions in the same cycle, the exception with the highest priority is always handled first. When any exception is taken, the DIVA checker fixes the errant instruction with the correct value (returned by either the CHKcomp or CHKcomm pipeline), flushes the processor pipeline, and then restarts the DIVA checker and processor core.

It is crucial that the DIVA checker be able to correct whatever condition resulted in a DIVA exception. If the DIVA checker were not able to correct a particular exccption condition, it would not be able to guarantee program forward progress in the presence of a permanent core fault (e.g., a design error or stuck-at fault). As shown in Figure 3 , all exception conditions are corrected. In fact, the DIVA checker is sufficiently robust that it can completely take over execution of the program in the event of a total core failure. However, its performance would be very poor, especially if it had to rely on the watchdog timer to expire before starting each instruction.

There is slightly different handling of the watchdog timer exception. When the watchdog timer expires, the DIVA checker fetches the next instruction to execute and injects it into the DIVA pipe with zero value inputs and outputs. The checker then restarts the processor. The CHKcomm and CHKcomp pipelines will correct these operands and results as incorrect values are detected, eventually completing execution of the stalled instruction.

\subsection{Working Examples}

Figure 4 shows two examples of the DIVA checker in operation. In Figure 4a, an AGEN operation produces an incorrect result that it forwards to a LD operation. The CHKcomp pipeline detects the incorrect result in the CMP stage and then declares a CHKcomp exception which corrects the result of the AGEN operation, allowing it to retire three cycles later.

Figure $4 \mathrm{~b}$ shows the operation of the DIVA checker in the event of a catastrophic core processor failure. In this example the core is not attempting to retire instructions, thus the DIVA checker must completely execute each instruction. The example starts out with a watchdog timer reset that forces insertion of the next instruction with zero value inputs and outputs. The instruction first detects in the CHKcomm pipeline that its inputs are incorrect which results in a CHKcomm exception that fixes the inputs. Next, the CHKcomp pipeline detects that the result is incorrect which declares a CHKcomp exception that fixes the result. Finally, the instruction completes without an exception and retires its results to the architected register file.

\subsection{Verification of the DIVA Checker}

Paramount to the success of dynamic verification is a functionally correct and electrically robust DIVA checker implementation. It had better work correctly all the time, 


\begin{tabular}{|c|c|c|c|}
\hline $\begin{array}{l}\text { DIVA } \\
\text { Exception }\end{array}$ & $\begin{array}{l}\text { Exception } \\
\text { Priority }\end{array}$ & $\begin{array}{l}\text { Recovery } \\
\text { Mechanism }\end{array}$ & $\begin{array}{l}\text { How the Exception } \\
\text { is Corrected by DIVA }\end{array}$ \\
\hline $\begin{array}{l}\text { Watchdog Timer } \\
\text { Expiration }\end{array}$ & 0 (highest) & $\begin{array}{l}\text { 1) reset DIVA pipes } \\
\text { with next inst } \\
\text { 2) restart DIVA pipes @ PC } \\
\text { 3) flush core, restart @ NPC }\end{array}$ & $\begin{array}{l}\text { Watchdog exception } \\
\text { jumpstarts DfVA checker } \\
\text { and core at next instruction }\end{array}$ \\
\hline $\begin{array}{l}\text { CHKcomm } \\
\text { (register value) }\end{array}$ & 1 & $\begin{array}{l}\text { 1) reset DIVA pipes } \\
\text { with correct register input } \\
\text { 2) restart DIVA pipes @ PC } \\
\text { 3) flush core, restart @ NPC }\end{array}$ & $\begin{array}{l}\text { CHKcomm RD stage register } \\
\text { value (always correct) is injected } \\
\text { into DIVA verification }\end{array}$ \\
\hline $\begin{array}{l}\text { CHKcomm } \\
\text { (memory value) }\end{array}$ & 2 & $\begin{array}{l}\text { 1) reset DIVA pipes } \\
\text { with correct memory input } \\
\text { 2) restart DIVA pipes @ PC } \\
\text { 3) flush core, restart @ NPC }\end{array}$ & $\begin{array}{l}\text { CHKcomm RD stage memory } \\
\text { value (always correct) is injected } \\
\text { into DIVA verification }\end{array}$ \\
\hline CHKcomp & 3 (lowest) & $\begin{array}{l}\text { 1) reset DIVA pipes } \\
\text { with correct register result } \\
\text { 2) restart DIVA pipes @ PC } \\
\text { 3) flush core, restart @ NPC }\end{array}$ & $\begin{array}{l}\text { CHKcomp EX' result (correct } \\
\text { if no other exceptions) is } \\
\text { injected into DIVA verification }\end{array}$ \\
\hline
\end{tabular}

Figure 3. Fault Handling in the DIVA Checker.

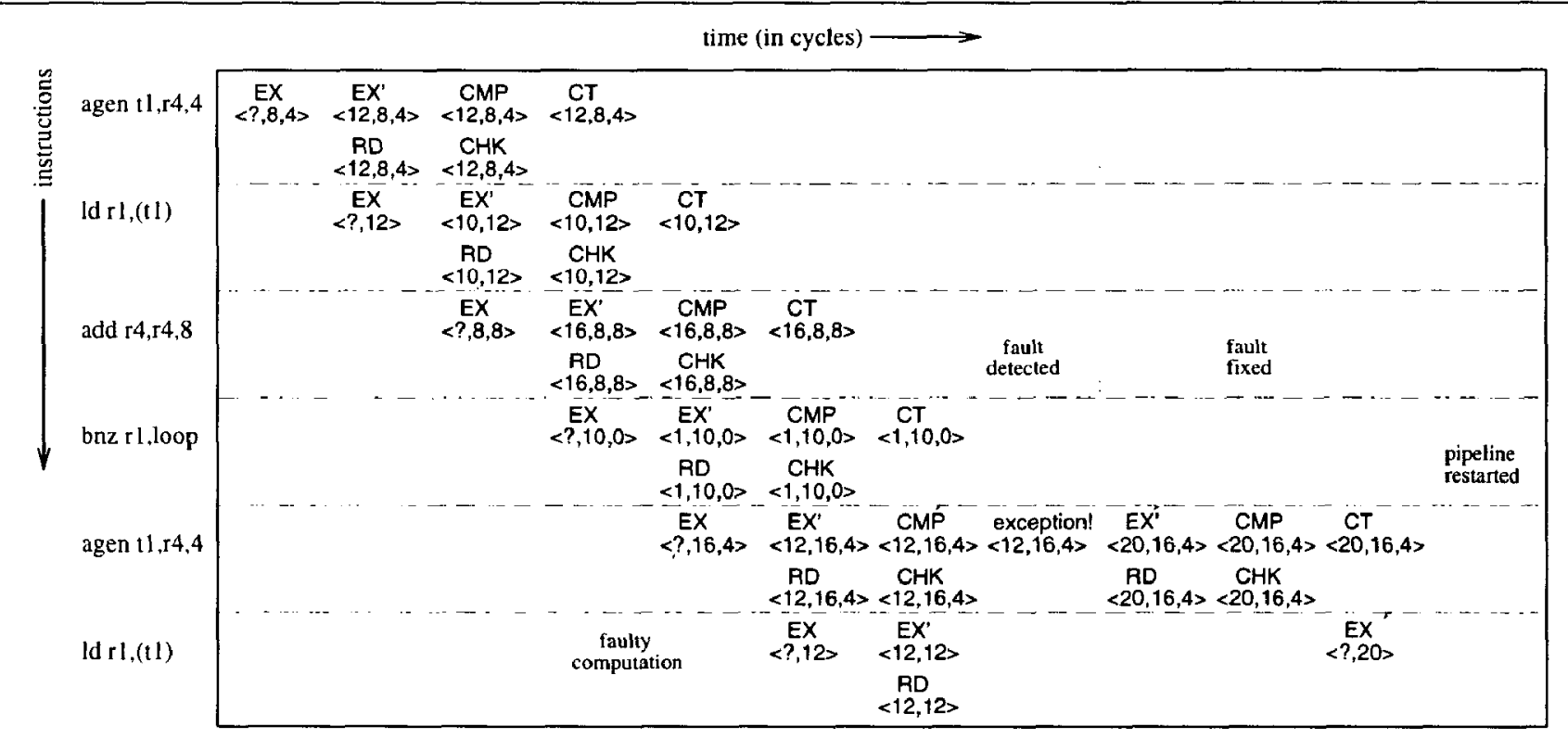

a)

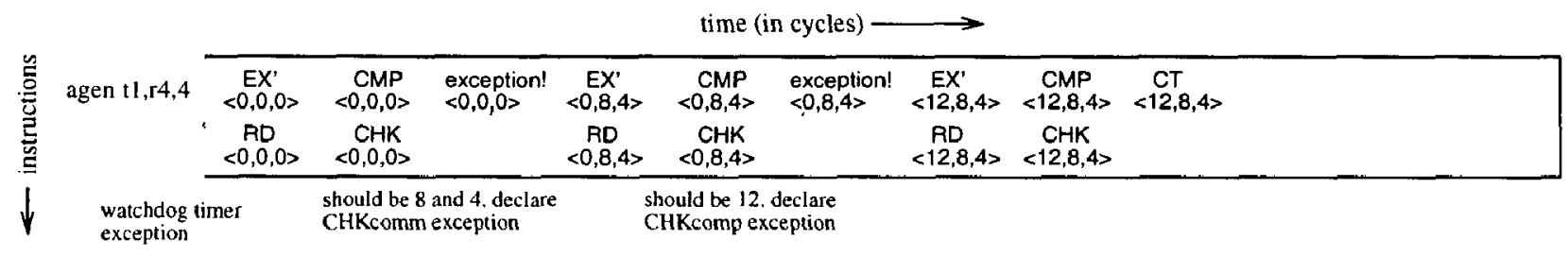

b)

Figure 4. Example Operation of the DIVA checker. Two working pipeline examples are shown in Figures a) and b). In the pipeline diagrams, program execution runs from top to hottom, the instruction executed is shown to the left of the pipeline. Time runs from left to right; instructions list which pipeline stage they are in for each cycle they are active. Below the pipeline stage designators are listed the one output and two input values for each instruction. The vertical bars represent declarations of DIVA checker exceptions. 


\begin{tabular}{|l||l|r|r|r|r|r|r|r|r|}
\hline Program & Input & $\begin{array}{c}\text { \# instr } \\
\text { fwd (M) }\end{array}$ & $\begin{array}{c}\text { \# instr } \\
\text { exec (M) }\end{array}$ & $\begin{array}{c}\text { \% ld } \\
\text { exe }\end{array}$ & $\begin{array}{r}\text { \% st } \\
\text { exe }\end{array}$ & $\begin{array}{c}\text { Base } \\
\text { CPI }\end{array}$ & $\begin{array}{r}\text { RUU } \\
\text { Occ }\end{array}$ & $\begin{array}{c}\text { Mem } \\
\text { Util }\end{array}$ & $\begin{array}{c}\text { BP } \\
\text { Acc }\end{array}$ \\
\hline \hline compress & ref.in & 0 & 93 & 26.7 & 9.4 & 0.60 & 84.2 & 0.41 & 90.2 \\
GCC & 1stmt.i & 100 & 100 & 24.6 & 11.5 & 0.64 & 25.5 & 0.32 & 85.4 \\
go & 2stone9.in & 100 & 100 & 30.7 & 8.2 & 0.61 & 23.3 & 0.42 & 76.1 \\
ijpeg & vigo.ppm & 100 & 100 & 18.5 & 5.6 & 0.38 & 132.0 & 0.39 & 88.6 \\
li & boyer.lsp & 100 & 100 & 25.8 & 15.1 & 0.47 & 52.9 & 0.44 & 93.1 \\
perl & scrabble.pl & 100 & 100 & 22.7 & 12.2 & 0.48 & 55.0 & 0.39 & 93.7 \\
\hline hydro2D & hydro2D.in & 100 & 100 & 20.7 & 8.7 & 0.46 & 106.4 & 0.37 & 96.3 \\
tomcatv & tomcatv.in & 100 & 100 & 20.4 & 8.7 & 0.42 & 50.2 & 0.40 & 95.6 \\
turbo3D & turbo3D.in & 100 & 100 & 23.6 & 16.2 & 0.38 & 149.7 & 0.42 & 94.9 \\
\hline
\end{tabular}

Table 1. Program statistics for the baseline architecture.

otherwise, it may impair correct operation of the processor core. Moreover, the cost of DIVA checker verification should be lower than the cost of verifying a traditional processor design, otherwise, there is no overall gain to the employing dynamic verification. It is difficult to quantitatively assess the ease (or difficulty) in building a correct DIVA checker in a paper design such as this. To accurately assess these costs would require the construction of a real DIVA checker in VLSI. In lieu of this level of detail, we describe the attributes of the DIVA checker design that we feel will lend the approach to high-quality and low-cost functional and electrical verification.

Simple: The DIVA checker is inherently simpler than a traditional processor core. It contains only the mechanisms necessary to check the function of the program, and it lacks all of the mechanisms used to speed computation, e.g., predictors, renamers, dynamic schedulers, etc. In addition, the pre-computed inputs and outputs from the core processor eliminate the inter-instruction dependencies and stall conditions that complicate traditional high-performance pipeline designs.

Latency-Insensitive: With sufficient buffering of speculative core results, the latency of the DIVA checker will not impact core processor performance. As a result, wide and deeply pipelined implementations are possible. These designs will permit checker implementations with large timing margins and large (and slow) transistors, affording the checker high resistance to transient faults and natural radiation interference. Since there are few dependencies between instructions, widening or lengthening the DIVA pipeline is quite straightforward.

Reusable: The DIVA checker design is more reusable than traditional processor cores, making it possible to leverage correctness established in previous designs. Since the checker sits at retirement, new designs need only scale with the retirement bandwidth of the new core it is checking. Retirement bandwidth scales very slowly from generation to generation, any additional bandwidth requirements can be accommodated by simply lengthening or widening the DIVA checker pipelines.

In addition to these attributes, we are currently investigating formal verification of the DIVA checker. The DIVA checker resembles a simple in-order processor with little microarchitectural state and few inter-instruction dependencies - properties that simplify formal verification [8, 24]. We believe the DIVA checker will also lend itself to formal verification, making it possible to formally verify large complex microarchitectures by only verifying the correctness of the DIVA checker.

\section{Experimental Evaluation}

In this section, we examine the impact of dynamic verification on processor core performance. Core slowdowns are measured, using detailed timing simulation for DIVA checkers with varied resource configurations, checker latency, and fault rates.

\subsection{Methodology}

The simulators used in this study are derived from the SimpleScalar/Alpha 3.0 tool set [9], a suite of functional and timing simulation tools for the Alpha AXP ISA. The timing simulator executes only user-level instructions, performing a detailed timing simulation of an aggressive 4way dynamically scheduled microprocessor with two levels of instruction and data cache memory. Simulation is execution-driven, including execution down any speculative path until the detection of a fault, TLB miss, or branch misprediction.

To perform our evaluation, we collected results for nine of the SPEC95 benchmarks [28]. All programs were compiled on a DEC Alpha AXP-21 164 processor using the DEC $\mathrm{C}$ and Fortran compilers under OSF/1 V4.0 operating system using full compiler optimization ( -04 -ifo). Table 1 shows the data set we used in gathering results for each program, the number of instructions that were executed (fast forwarded) before actual simulation began, and the number of instructions simulated for each program (up to $100 \mathrm{mil}$ lion). Also shown are the percent of dynamic instructions that were loads and stores, the baseline machine CPI, the average number of entries in the instruction window (RUU), the fraction of time the memory ports were in use, and the branch predictor accuracy for each program.

\subsection{Baseline Architecture}

Our baseline simulation configuration models a future generation out-of-order processor microarchitecture. We've 
selected the parameters to capture underlying trends in microarchitecture design. The processor has a large window of execution; it can fetch and issue up to 4 instructions per cycle. It has a 256 entry re-order buffer with a 64 entry load/store buffer. Loads can only execute when all prior store addresses are known. In addition, all stores are issued in program order with respect to prior stores. There is an 8 cycle minimum branch misprediction penalty. The processor has 4 integer ALU units, 2-load/store units, 2-FP adders, 1 -integer MULT/DIV, and 1-FP MULT/DIV. The latencies are: ALU 1 cycle, MULT 3 cycles, Integer DIV 12 cycles, FP Adder 2 cycles, FP Mult 4 cycles, and FP DIV 12 cycles. All functional units, except the divide units, are fully pipelined allowing a new instruction to initiate execution each cycle.

The processor we simulated has a $32 \mathrm{k}$ 2-way setassociative instruction and data caches. Both caches have block sizes of 32 bytes. The data cache is write-back, writeallocate, and is non-blocking with 2 ports. The data cache is pipelined to allow up to 2 new requests each cycle. There is a unified second-level $512 \mathrm{k}$ 4-way set-associative cache with 32 byte blocks, with a 10 cycle cache hit latency. If there is a second-level cache miss it takes a total of $60 \mathrm{cy}$ cles to make the round trip access to main memory. We model the bus latency to main memory with a 10 cycle bus occupancy per request. There is a 32 entry 8-way associative instruction TLB and a 32 entry 8-way associative data TLB, each with a 30 cycle miss penalty.

\subsection{DIVA Checker Baseline Architecture}

The DIVA checker in all experiments is a four instruction wide pipeline that instructions enter when they have completed and are the oldest instruction in the machine that has not yet entered the DIVA checker pipeline. Instructions are processed in-order, any instruction that stalls causes later instructions to also stall. In the baseline configuration, the CHKcomp pipeline latency is one cycle longer than the functional unit it checks (for the result comparison). It is assumed that there is a CHKcomp pipeline for each of the functional units, as a result, there are no structural hazards introduced. The baseline CHKcomm pipeline takes two cycles (for RD and CHK) unless there are structural hazards in accessing register file and cache ports. In the baseline checker architecture, the RD stage competes with the core processor for four architected register file ports and two cache ports, with priority given to the DIVA checker accesses. The core processor only accesses the architected register file when an operand is not found in the physical register file (i.e., it is not in flight). Re-order buffer entries are not deallocated until instructions exit the commit (CT) stage of the pipcline, after the DIVA checker verifies the operation. The watchdog timer countdown is reset to 60 cycles (the round trip latency to memory) whenever an instruction commits.

\subsection{DIVA Checker Resource Requirements}

The DIVA checker requires register file and data cache storage bandwidth to verify program communication. (Dur-

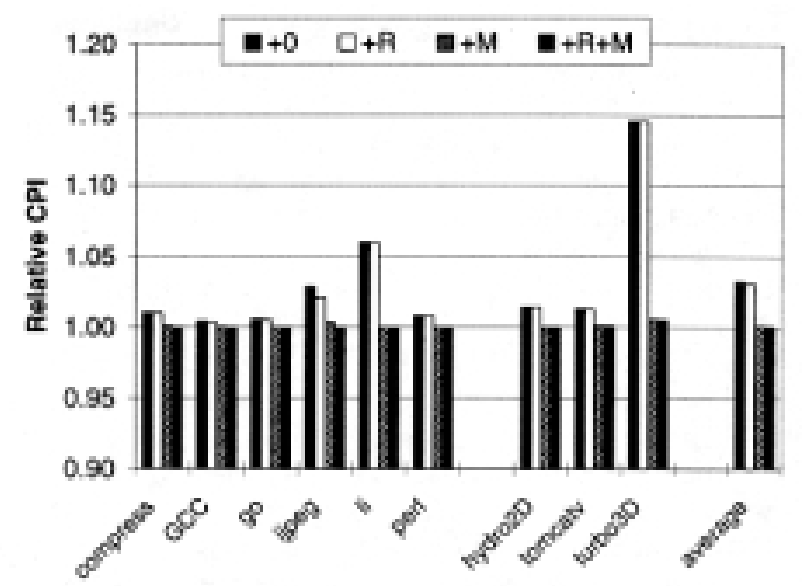

Figure 5. DIVA Checker Impact on Core Processor Performance for Varied Register File and Cache Bandwidth. All performance numbers are normalized to the CPI of an unchecked core processor. Results are shown with no extra register file or cache ports $(+O)$, with 4 extra register file ports $(+R)$, with one extra memory port $(+M)$, and with 4 extra register file ports and one extra memory port $(+R+M)$.

ing the $\mathrm{RD}$ stage of the $\mathrm{CHK}$ comm pipeline all register and memory communication is re-executed in program order.) Without dedicated ports into the architected register file and data cache, the DIVA checker must compete for bandwidth with the core processor. This competition can create structural hazards which can slow core processing.

In Figure 5, we show the impact of DIVA checker resources on core processor performance. All performance numbers are normalized to the CPI of an unchecked core processor. Results are shown with no extra register file or cache ports $(+0)$, with 4 extra register file ports dedicated to the DIVA checker $(+R)$, with one extra dedicated memory port $(+M)$, and with 4 extra register file ports and one extra memory port $(+R+M)$.

Even without extra resources, i.e., experiment $(+0)$, the cost of employing the DIVA checker is quite low. Without any extra register file or memory ports, average program slowdown was only $3 \%$. In general, there was a high correlation between pipeline utilization (e.g., branch prediction accuracy, RUU occupancy, and memory port utilization) and slowdown. When the processor pipeline is efficiently utilized, any additional DIVA checker register file and cache accesses create structural hazards that slow core processing. Turbo3D had the largest slowdown of $14 \%$ without additional resources. This benchmark is highly efficient, it has high branch predictor accuracy, high RUU occupancy and high memory utilization. GCC and and GO, on the other hand, have poor branch prediction and thus poor pipeline utilization; additional DIVA checker resource usage has little impact on the core processor performance of these programs.

Experiment $(+R)$ adds four more read ports to the architected register file for use by the DIVA checker CHKcomm pipeline. These additional register ports eliminate most 
structural hazards into the architected register file. This change had little impact on overall performance (at most an improvement of $0.9 \%$ for $H y d r o 2 D$ ). Since many of the register accesses are satisfied by the physical register filc (which has its own access ports), there appears to be sufficient bandwidth left into the architected register file for DIVA checker accesses.

Experiment $(+M)$ adds one more read port to the data cache for use by the DIVA checker CHKcomm pipeline. This additional cache port eliminates nearly all structural hazards into the data cache. Adding this port has a noticeable impact on core processor performance. Most core processor performance impacts are eliminated and overall slowdown drops to only $0.1 \%$.

Finally, in experiment $(+R+M)$, four register ports and two memory ports are added for DIVA checker use. With an additional memory port, the extra register file ports provide little benefit, and overall slowdown drops to $0.03 \%$.

\subsection{Effects of Increased DIVA Checker Latency}

In an effort to build an electrically robust implementation of the DIVA checker, it may be necessary to construct it with large timing margins and large transistors (to resist noise and tolerate natural radiation interference, respectively). The most straightforward approach to achieve this is to deeply pipeline the DIVA checker. Decply pipclining the DIVA checker, however, will increase its latency which can delay retirement of instructions. These delays may cause congestion in the instruction window, effectively reducing the instruction window size and amount of ILP that can be exploited. Figure 6 shows the impact of DIVA checker latency of core processor performance. All performance numbers are normalized to the CPI of a DIVA checker configuration with no extra register file or cache port resources, i.e., experiment $(+O)$ from Figure $5 .{ }^{4}$ Results are shown for a checker with two times as many stages $(2 x)$, and a checker with four times as many stages $(4 x)$

Overall, the impact of checker latency on core performance is quite low. At two times the latency slowdowns increase by $0.7 \%$, and with four times the checker latency, slowdowns only increase by $0.8 \%$. Even the programs with high branch predictor accuracy. e.g., Hydro2D, cannot get sufficiently far ahead of the execution core to keep the instruction window full, as a result, adding extra checker latency has little impact on core performance.

\subsection{Effects of DIVA Checker Exceptions}

In the event that the DIVA checker detects a failed computation or communication, it will declare a DIVA exception and reset the checker and processor core. The performance penalty for exception handling is quite large, at least 8 cycles for the experiments simulated, more if the faulty instruction takes a while to reach retirement. To gauge the performance impact of DIVA exceptions, we ran experiments with random exceptions injected at random times but

\footnotetext{
${ }^{4}$ We compare to this configuration because it was the worst performing DIVA checker configuration, thus it will have the most instruction window congestion to start with and be most sensitive to DIVA checker latency.
}

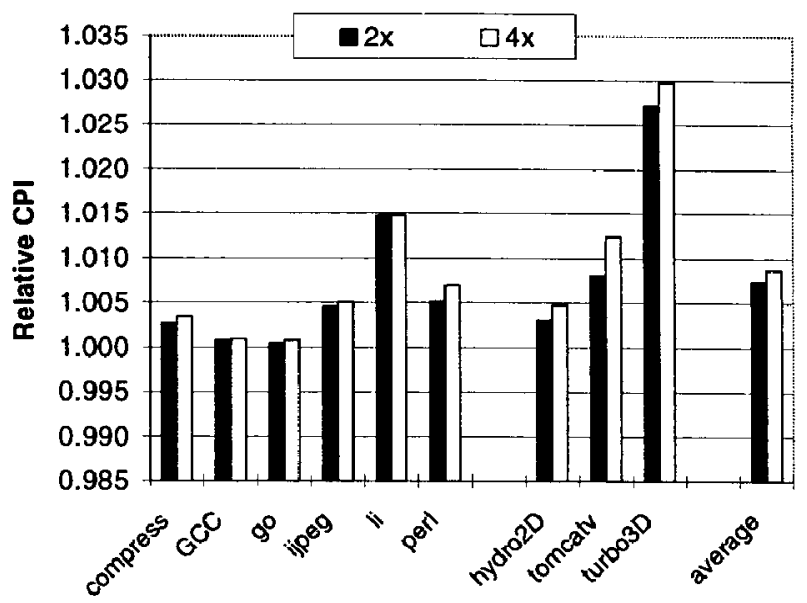

Figure 6. DIVA Checker Impact on Core Processor Performance with Varied DIVA Checker Latency. All performance numbers are normalized to the CPI of the baseline DIVA checker configuration $(+0)$. Results are shown for a checker with two times the latency $(2 x)$, and a checker with four times the latency $(4 x)$.

with a fixed exception interval (in core processor cycles). The results are shown in Figure 7. All performance numbers are normalized to the CPI of the baseline DIVA checker configuration $(+0)$. Results are shown on a log scale for exception rates of one per one million core processor cycles $(I M)$, one per 1000 processor cycles $(l k)$, and one every processor cycle $(I)$. The experiment labeled (Core Lock) examined the performance impacts of a catastrophic core failure in which the core is no longer attempting to commit instructions, consequently, instructions are not fetched until the watchdog timer counter expires (once every 60 cycles).

At an exception rate of one exception every one million cycles (an average of one exception every $2 \mathrm{msec}$ on a 500 $\mathrm{MHz}$ processor), there was virtually no impact on core processor performance. At intervals of 1000 cycles (an average of one exception every 2 usec on a $500 \mathrm{MHz}$ processor), impacts were higher but still small, with an overall slowdown of only $2.6 \%$. With an exception every cycle (as would be the casc for a catastrophic corc processor failure), performance impacts rise dramatically. Overall, performance is $\frac{1}{14}$ th normal speed. In this experiment, the DIVA checker is completely executing the program. When the core processor is locked up, performance is quite low, on average $\frac{1}{120}$ th the performance of the unchecked core. In Section 5 , we suggest a simple change to detect this case and provide more graceful degradation in processor performance.

\subsection{Discussion}

Core processor slowdowns (due to structural hazards, checker latency, and exception handling) are not the only costs associated with the DIVA checker, other costs include silicon area, power consumption, among others. While it is difficult to gauge these costs without building an actual DIVA checker implementation, we feel overall DIVA checker costs should be low for at least two reasons. 


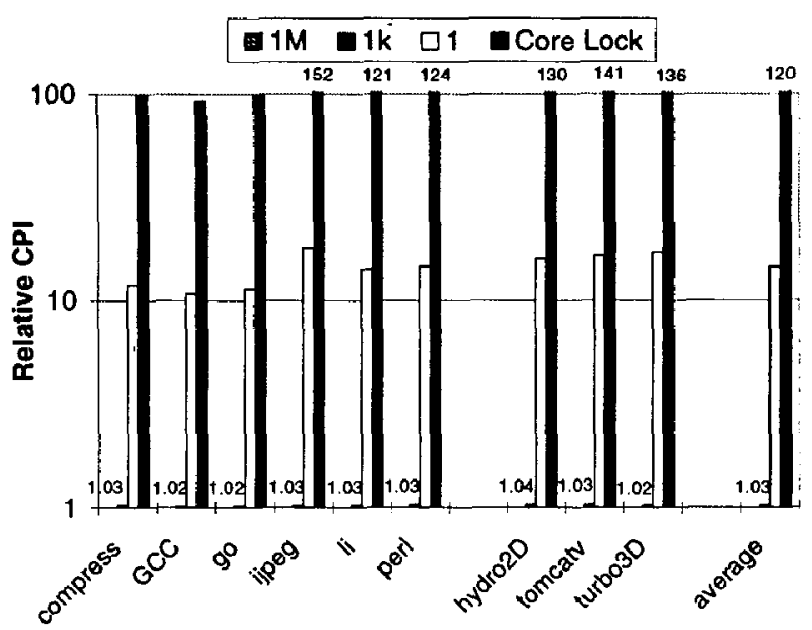

Figure 7. Performance Impact of Varied Exception Rates. All performance numbers are normalized to the CPI of the baseline DIVA checker configuration $(+O)$. Results are shown on a log scale for exception rates of one per one million core processor cycles $(I M), 1000$ processor cycles $(I k)$. and every processor cycle $(l)$. The experiment labeled (Core Lock) examines the performance of a catastrophic core failure in which the core is no longer attempting to commit instructions. Bar values are shown for the (Core Lock) and ( $1 k)$ experiments.

First, the DIVA checker only replicates the part of core pertaining to actual function. All core processing structures related to program performance (e.g., predictors and schedulers) need not be represented in the DIVA checker. In addition, the DIVA checker design is a very simple pipetine. It uses in-order instruction processing and has few inter-instruction dependencies. These advantages make the DIVA checker significantly simpler than the core processor pipeline, and they should serve to reduce its size and cost.

Second, the CHKcomp pipeline can be implemented with simple, area-efficient algorithms. For example, if the core uses a carry-select carry-lookahead adder for fast addition, the DIVA checker can instead use a pipelined ripple carry adder to reduce implementation costs. The pipeline will be slower, but as shown earlier, core performance is mostly insensitive to DIVA checker latency, making this a good tradeoff.

Ultimately, a more quantitative assessment of DIVA checker area and power costs will have to be made, first through circuit level simulation and later through an actual silicon implementation. We are currently working toward these goals.

\section{Related Work}

The idea of dynamic verification at retirement was inspired by Breach's Multiscalar processor simulator [7]. During the programming and verification of this very complex simulator, a functional verification stage was added at retirement. The approach was sufficiently robust that it could mask functional bugs that were introduced during the simulator's development permitting its use for performance analysis before it was fully debugged. The DIVA checker works in precisely the same manner, but for a hardware system.

Rotenberg's AR-SMT processor [22] employs a timeredundant execution technique that permits an SMT processor to tolerate some transient errors. We borrow on this work; the DIVA checker leverages the idea of using an unreliable processor's results to speed the execution of instruction checking. We improve upon Rotenberg's work in a number of ways. Rotenberg's approach checks all aspects of execution, include program function and the mechanisms used to optimize program performance. The DIVA checker, on the other hand, only checks program function, permitting a simpler checker implementation. While Rotenberg's approach only detects some transient errors, a functionally correct and electrically robust DIVA checker can recover all permanent and transient core processor faults.

A number of fault-tolerant processor designs have been proposed and implemented, in general, they employ redundancy to detect and/or correct transient errors. IBM's G4 processor [27] is a highly reliable processor design similar to the design in this paper in that it checks all instructions results before committing them to architected state. Checking is accomplished by fully replicating the processor core. An R-Unit is added to compare all instruction results, only permitting identical results to commit. If a failure in the processor is detected, it is addressed at the system level through on-line reconfiguration. The ERC32 is a reliable SPARC compatible processor built for space applications [12]. This design augments the microarchitecture with parity on all register and memory cells, some self-checking control logic, and control flow checking. In the event a fault is detected, a software interrupt is generated and the host program initiates recovery.

Unlike these designs, the DIVA checker can keep costs lower by only checking the function of the program computation. The G4 and ERC32 designs check both the function of the program and the mechanisms used to optimize program performance. This results in more expensive checkers, typically 2 times as much hardware in the core processor. Additionally, the DIVA checker can detect design errors. Simple redundant designs cannot detect design errors if the error is found in each of the redundant components.

Tamir and Tremblay [29] proposed the use of micro rollback to recover microarchitecture state in the event of a detected fault. The approach uses FIFO queues to checkpoint a few cycles of microarchitectural state. In this work, we use a similar parallel checking approach, but employ a global checking strategy to reduce checker cost. In addition, we use the existing control speculation mechanism to restore correct program state.

\section{Other DIVA Applications}

The next logical step in this work is to explore ways to further exploit fault tolerance in the DIVA core. In this section, we present two other application of dynamic verification. 


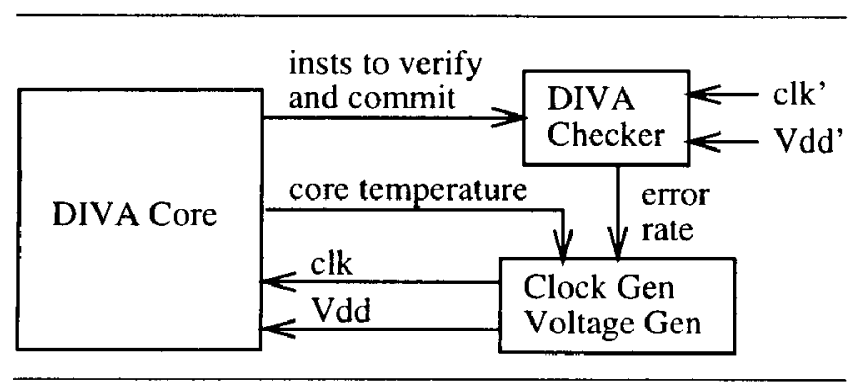

Figure 8. A Self-Tuned System.

\subsection{Self-Tuned Systems}

The techniques used to provide reliability in VLSI logic implementations are very conservative. Systems are designed with frequency and voltage margins that ensure reliable operation in even the most adverse environments (e.g., high temperature, high clock skew, slow transistors). This is onc of the reasons why hobbyists can ovcrelock [20] production parts for more performance. It should be possible to use the DIVA checker to reclaim much of the power and performance consumed by operating margins.

In a self-tuned system [19], clock frequency and voltage levels are tuned to the system operating environment, e.g., temperature. The approach minimizes timing and voltage margins which can improve performance and reduce power consumption. Using the DIVA checker, a self-tuned system could be constructed by introducing a voltage and frequency control system into the processor, as shown in Figure 8. The control system decreases voltage and/or increases frequency while monitoring system temperature and error rates until the desired system performance-power characteristics are attained. If the control system over steps the bounds of correct operation in the core, the DIVA checker will correct the error, reset the core processor, and notify the control system. To ensure correct operation of the DIVA checker, it is sourced by a fixed voltage and frequency that ensures reliable operation under all operating conditions.

\subsection{Highly Available Designs}

As shown earlier, if the core processor completely fails and no longer attempts to retire instructions, processor performance will be severely impacted. Without any instruction retiring, the DIVA checker will have to wait for the watchdog timer to timeout before an instruction can be retired and the next instruction fetched. As a result, one instruction will complete for each interval of the watchdog timer, one per 60 clock cycles in the experiments presented.

It would be relatively straightforward to detect the case where the core processor was no longer contributing to the program execution. For example, a counter could record the number of watchdog timer exceptions with no intervening instruction retirements. If a suitable threshold was reached, it could be assumed that the core processor has failed and the DIVA checker could take over execution of the program. A simple change to the DIVA design would permit it to fetch and execute instruction much quicker, simply by per-

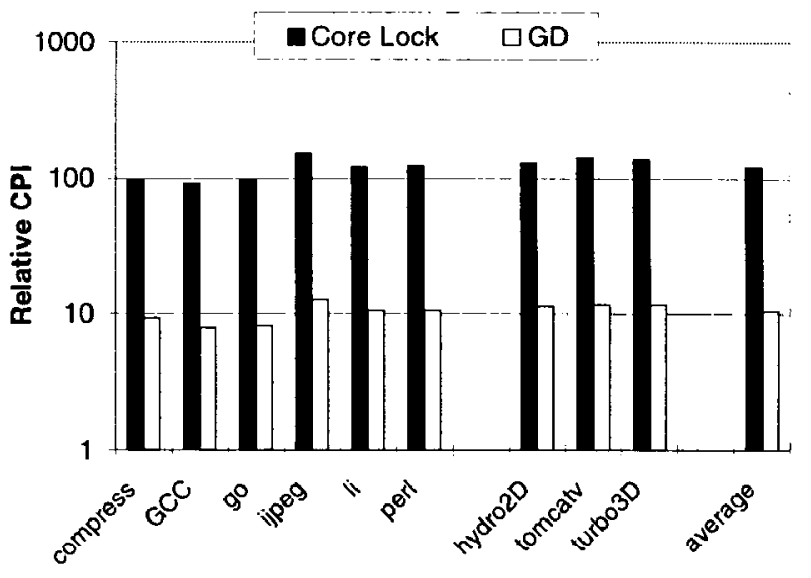

Figure 9. Graceful Degradation in the Presence of a Catastrophic Core Processor Failure. All performance numbers are normalized to the CPI of an unchecked core processor. Results are shown on a log scale for the baseline DIVA checker with a locked core (Core Lock), and for a DIVA checker with graceful degradation $(G D)$.

mitting it to execute the next instruction without having to wait for the watchdog timer to expire.

As shown in experiment $(G D)$ in Figure 9, this graceful degradation approach improves processor performance considerably in the event of a catastrophic core processor failure. Program performance degrades to only $\frac{1}{10}$ th the performance of the original working core processor. This is a marked improvement over the (Core Lock) experiment, in which the core processor locks and instructions proceed at watchdog timer exceptions, an average of $\frac{1}{120}$ th the speed of the working core processor. The graceful degradation mode also outperforms a core processor that is still retiring instructions but always incorrect results, i.e., experiment ( $I$ ) from Figure 7, with an overall performance of $\frac{1}{14}$ th the speed of the working core. In this case, the DIVA checker benefits from not having to wait for the core processor pipeline to first execute the instruction incorrectly.

In addition, the availability of a system using the DIVA checker could be further improved by replicating the DIVA checker to detect errors within its own circuitry, or by applying triple modular redundancy (TMR) [26] to detect and correct permanent faults in the DIVA checker.

\section{Conclusions}

Many reliability challenges confront modern microprocessor designs. Functional design errors and electrical faults can impair the function of a part, rendering it useless. While functional and electrical verification can find most of the design errors, there are many examples of non-trivial bugs that find their way into the field. Concerns for reliability grow in deep submicron fabrication technologies due to increased noise-related failure mechanisms, natural radiation interference, and more challenging verification due to increased design complexity. 
To counter these reliability challenges, we introduced dynamic verification, a technique that adds a functional checker to the retirement phase of a processor pipeline. The functional checker ensures that all core processor computation is correct, and if not, the checker fixes the errant computation, and restarts the core processor using the processor's speculation recovery mechanism. Dynamic verification focuses the verification effort into the checker unit, whose simple and flexible design lends itself to functional and electrical verification. Using this approach, the core processor carries no burden of correctness or any requirement for forward progress. The DIVA checker architecture was presented as a checker design optimized for simplicity and low cost.

Detailed timing-simulation based analyses were used to examine the performance impact of the DIVA checker. Overall, performance impacts, even for resource-frugal designs, were small. For the least expensive design analyzed, the DIVA checker only slowed the core processor an average of $3 \%$. With the addition of a single memory port for use by the DIVA checker, slowdowns were negligible. Also, increased DIVA checker latency had only a small impact on core processor performance. The DIVA checker can tolerate very high fault rates without significantly impacting processor core performance. At rates of one fault per 1000 processor cycles, the core processor only slowed by $2.6 \%$; at intervals of $1 \mathrm{M}$ cycles, performance impacts were negligible.

Finally, other applications of dynamic verification were proposed. A self-tuned clock and voltage scheme was proposed in which dynamic verification is used to reclaim frequency and voltage margins. A highly available checker design was also proposed. The design provides more graceful degradation in system performance in the event of a total core processor failure.

We feel that dynamic verification holds significant promise as a means to address the cost and quality of verification for future microprocessors, while at the same time creating opportunities for faster, cooler, and simpler designs. The next step in this work is to better quantify the benefits and costs of dynamic verification, and to further develop and refine other applications of the fault tolerant processor core.

\section{References}

[1] A. Aharon. Test program generation for functional verification of powerpc processors in IBM. In Proceedings of the 32nd ACM/IEEE Design Automation Conference, pages 279285, June 1995.

[2] R. Anglada and A. Rubio. An approach to crosstalk effect analyses and avoidance techniques in digital CMOS VLSI circuits. International Journal of Electronics, 6(5):9-17, 1988.

[3] J. Bockhaus, R. Bhatia, M. Ramsey, J. Butler, and D. Ljung. Electrical verification of the HP PA- 8000 processor. HewlettPackard Journal, Aug. 1997.

[4] M. Bohr. Interconnect scaling - the real limiter to highperformance ULSI. In Proceedings of the International Elec. tron Devices Meeting, pages 241-244, Dec. 1995.
[5] P. Bose and T. Conte. Performance analysis and its impact on design. IEEE Computer, 31(5):41-49, May 1998.

[6] P. Bose, T. Conte, and T. Austin. Challenges in processor modeling and validation. IEEE Micro, pages 2-7, June 1999.

[7] S. Breach. Design and Evaluation of a Multiscalar Processor. PhD thesis, University of Wisconsin, Madison, 1999.

[8] J. Burch and D. Dill. Automatic verification of pipelined microprocessors control. Computer Aided Verification, pages 68-80, 1994.

[9] D. C. Burger and T. M. Austin. The simplescalar tool set, version 2.0. Technical Report CS-TR-97-1342, University of Wisconsin, Madison, June 1997.

[10] B. Colwell. Maintaining a leading position. IEEE Computer, pages $47-48$, Jan. 1998 .

[11] J. Z. et al. IBM experiments in soft fails in computer electronics. IBM Journal of Research and Development, 40(1):318, Jan. 1996.

[12] J. Gaisler. Evaluation of a 32-bit microprocessor with built-in concurrent error detection. In Proceedings of the 27th FaultTolerant Computing Symposium, June 1997.

[13] R. Grinwald. User defined coverage - a tool supported methodology for design verification. In Proceedings of the 35nd ACM/IEEE Design Automation Conference, pages 1-6, June 1998.

[14] G. Grohoski. Reining in complexity. IEEE Computer, pages 47-48, Jan. 1998.

[15] W. Hunt. Microprocessor design verification. Journal of Automated Reasoning, 5(4):429-460, Dec. 1989.

[16] Statistical analysis of floating point flaw in the Pentium processor. Intel Corporation, Nov. 1994.

[17] W. J. Self-Checking Circuits and Applications. New York: North-Holland, 1978.

[18] M. Kane. SGl stock falls following downgrade, recall announcement. PC Week, Sept. 1996.

[19] T. Kehl. Hardware self-tuning and circuit performance monitoring. In Proceedings of International Conference on Computer Design, 1993.

[20] B. Machrone. You too can be an overclocker. PC Magazine, 1999.

[21] T. May and M. Woods. Alpha-particle-induced soft errors in dynamic memories. JEEE Transactions on Electronic Devices, 26(2), 1979.

[22] E. Rotenberg. AR-SMT: A microarchitectural approach to fault tolerance in microprocessors. In Proceedings of the 29th Fault-Tolerant Computing Symposium, June 1999.

[23] P. Rubinfeld. Managing problems at high speed. IEEE Computer, pages 47-48, Jan. 1998

[24] J. Sawada. A table based approach for pipelined microprocessor verification. In Proceedings of the 9 th International Conference on Computer Aided Verification, June 1997.

[25] K. Seshan, T. Maloney, and K. Wu. The quality and reliability of Intel's quarter micron process. Intel Technology Journal, Sept. 1998

[26] D. Siewiorek and R. Swarz. The Theory and Practice of Reliable System Design. Digital Press, 1982.

[27] L. Spainhower and T. Gregg. G4: A fault-tolerant CMOS mainframe. In Proceedings of the 28th Fault-Tolerant Computing Symposium, June 1998.

[28] SPEC newsletter. Fairfax, Virginia, Sept. 1995

[29] Y. Tamir and M. Tremblay. High-performance fault tolerant VLSI systems using micro rollback. IEEE Transactions on Computers, 39(4):548-554, 1990.

[30] $\mathrm{M}$. Tremblay. Increasing work, pushing the clock. IEEE Computer, pages 47-48, Jan. 1998.

[31] N. Weste and K. Eshragian. Principles of Cmos VLSI Design: A Systems Perspective. Addison-Wesley Publishing Co., 1982.

[32] A. Wolfe. For Intel, it's a case of FPU all over again. EE Times, May 1997.

[33] J. Ziegler. Terrestrial cosmic rays. IBM Journal of Research and Development, 40(1):19-39, Jan. 1996. 